\title{
Thermal degradation and viscoelastic properties of Kevlar/Cocos nucifera sheath reinforced epoxy hybrid composites
}

\begin{abstract}
The aim of this research work is to develop high performance structural composites using Kevlar 29 (K) and Cocos nucifera sheath (CNS). The Kevlar and CNS laminates were fabricated by using hand lay-up method followed by hot pressing. The weight ratios of Kevlar/CNS are as follows 100/0 (S1), 75/25(S2), 50/50 (S3), 25/75 (S4), 0/100(S5). Thermal and viscoelastic properties of laminated composites were investigated as a function of temperature using thermogravimetric (TGA) and dynamic mechanical analyzer (DMA). The obtained results revealed that the thermal stability, char residue of S2 laminate was higher compared S3, S4 and S5 laminates. Moreover, S2 laminates showed comparable thermal stability with Kevlar/epoxy composites (S1). Differential scanning calorimetry (DSC) results revealed that hybrid composite (S2) offers a virtuous resistance or stability towards heat in the epoxy composites. Viscoelastic analysis results showed that the storage modulus (E') and loss modulus (E") of S2 composites were higher among the laminates due to improved interfacial interactions and effective stress transfer rate. Moreover, the damping of hybrid laminates (S2) almost closer to Kevlar/epoxy laminates (S1). Hence, it was observed that hybrid Kevlar/CNS composites (S2) can be efficiently utilized for advanced structural applications where rigidity, thermal stability along with renewability are prime requirements.
\end{abstract}

Keyword: Kevlar; Cocos nucifera sheath; Hybrid composites; Thermal analysis; Viscoelastic properties 